\title{
Health care costs associated with venous
} thromboembolism in selected high-risk \section{ambulatory patients with solid tumors undergoing chemotherapy in the United States}

\author{
This article was published in the following Dove Press journal: \\ ClinicoEconomics and Outcomes Research \\ II February 2013 \\ Number of times this article has been viewed
}

\author{
Alok A Khorana' \\ Mehul R Dalal ${ }^{2}$ \\ Jay $\operatorname{Lin}^{3}$ \\ Gregory C Connolly' \\ 'Division of Oncology/Hematology, \\ James PWilmot Cancer Center, \\ University of Rochester, Rochester, \\ NY, ${ }^{2}$ sanofi-aventis US, Bridgewater, \\ $\mathrm{NJ},{ }^{3}$ Novosys Health, Flemington, \\ $\mathrm{NJ}$, USA
}

\begin{abstract}
Background: This study examines venous thromboembolism (VTE)-associated resource utilization and real-world costs in ambulatory patients initiating chemotherapy for selected common high-risk solid tumors.

Methods: Health care claims data (2004-2009) from the IMS/PharMetrics ${ }^{\circledR}$ Patient-Centric database were collected for propensity score-matched adult cancer (lung, colorectal, pancreatic, gastric, bladder, or ovarian) patients initiating chemotherapy with VTE $(\mathrm{n}=912)$ and without VTE $(n=2736)$. Health care resource utilization (inpatient, outpatient, and outpatient prescription drug claims) and costs were compared between the two cohorts during the 12-month follow-up period after the index VTE event. Incremental costs were adjusted for demographic and clinical covariates.

Results: Cancer patients with VTE had approximately three times as many all-cause hospitalizations (mean 1.38 versus 0.55 per patient) and days in hospital (10.19 versus 3.37), and more outpatient claims (331 versus 206) than cancer patients without VTE (all $P<0.0001$ ). Cancer patients with VTE incurred higher overall all-cause inpatient costs (mean USD 21,299 versus USD 7459 per patient), outpatient costs (USD 53,660 versus USD 34,232 per patient), and total health care costs (USD 74,959 versus USD 41,691 per patient) than cancer patients without VTE (all $P<0.0001$ ). Total mean VTE-related health care costs were USD 9247 per patient over 12 months. Adjusted mean incremental all-cause health care costs of VTE were USD 30,538 per patient for cancer overall, ranging from USD 11,946 for gastric to USD 38,983 for pancreatic cancer.
\end{abstract}

Conclusion: VTE is associated with significant inpatient and outpatient resource utilization, and increased all-cause (in addition to VTE-related) health care costs among ambulatory cancer patients. Measures to prevent outpatient cancer-associated VTE may reduce health care utilization and costs in this population.

Keywords: cancer, venous thromboembolism, resource utilization, health care costs, cohort study

\section{Introduction}

Venous thromboembolism (VTE), which includes deep vein thrombosis and pulmonary embolism, is a common complication of cancer and cancer treatment. ${ }^{1-3}$ Patients with active malignancy are at 4-7-fold higher risk of symptomatic VTE than the general population, ${ }^{4,5}$ and this prothrombotic risk is further exacerbated by chemotherapy, hormonal therapy, and surgery. ${ }^{3,6-8}$ In addition, patients with cancer who develop VTE are at higher risk of recurrent thrombotic complications than noncancer patients
Correspondence: Alok Khorana

University of Rochester, James P Wilmot

Cancer Center, 60I Elmwood Avenue,

Box 704, Rochester, NY 14642, USA

Tel + I 5852734 I 50

Fax +I 5852734150

Email alok_khorana@urmc.rochester.edu 
with VTE. ${ }^{9,10}$ Despite the strong association of VTE with cancer, there is wide variation in risk, with rates ranging from $0.6 \%$ to $7.8 \%$ in population-based case-control studies..$^{9,11,12}$

Predisposing factors for VTE in cancer patients include tumor site, ${ }^{13-15}$ stage (advanced/metastatic cancer), ${ }^{13,14}$ patient-related factors (advanced age, comorbidity, prolonged immobilization), ${ }^{5,15,16}$ and treatment-related factors, such as specific antineoplastic agents and growth factors. ${ }^{5,17}$ Few studies have assessed the economic impact of VTE in cancer patients. The limited data available suggest that management of cancer-associated VTE is both resource-intensive and costly, ${ }^{18-20}$ partly due to the high frequency of VTE recurrence, complications of anticoagulant therapy, ${ }^{18}$ and length of hospital stay. ${ }^{18,21}$ A review of medical records of cancer patients who developed VTE between 1994 and 1997 indicated that the mean cost of hospitalization for initiation of anticoagulant therapy was USD 20,065 (2002 USD values). ${ }^{18}$ Little is known about the current real-world economic burden of VTE events in ambulatory cancer patients undergoing chemotherapy or how this might differ according to the site of the cancer. Therefore, the objective of this study was to examine all-cause and VTE-associated resource utilization and assess, from the US health payer's perspective, the incremental cost of VTE in a large, real-world, contemporary cohort of ambulatory patients initiating chemotherapy for six selected common solid tumors (lung, colorectal, pancreatic, gastric, bladder, and ovarian).

\section{Materials and methods}

\section{Data source}

This retrospective observational cohort study was based on health care data collected from the IMS/PharMetrics ${ }^{\circledR}$ Patient-Centric database (IMS Health Inc) for the period January 2004 to December 2009. This large database provides integrated enrolment, medical, and prescription claims information from more than 90 managed care organizations and Medicare, and represents the health services of over 58 million patients across the US. It includes both inpatient and outpatient diagnoses (in International Classification of Diseases, Ninth Revision, Clinical Modification [ICD-9-CM] format), and procedures (in Current Procedural Terminology, Fourth Edition, and Health Care Common Procedure Coding System formats), and outpatient prescription records. Additional data elements include patient demographics, health plan type, payer type, provider specialty, and health plan enrolment dates. The IMS/PharMetrics Patient-Centric database has been used extensively for health economics and health outcomes research in various therapy areas, including VTE. ${ }^{22}$ All patient records used in the study were deidentified in compliance with the Health Insurance Portability and Accountability Act of 1996, so this study was exempt from institutional review board overview.

\section{Patient selection}

Patients aged $\geq 18$ years with an inpatient diagnosis of malignant neoplasm of the lung (ICD-9-CM code 162.0, 162.2-162.5, 162.8, 162.9), pancreas (157.0-157.4, 157.8, 157.9), stomach (151.0-151.6, 151.8, 151.9), colon/rectum (153.0-154.3, 154.8), bladder (188.x), or ovary $(183.0,183.2-183.5,183.8$, 183.9), and who received cytotoxic chemotherapy between January 2005 and December 2008 (index event identification period) were selected. Use of chemotherapy was indicated by pharmacy claims with relevant National Drug Code or Healthcare Common Procedure Coding System drug codes. For inclusion in the study, patients were additionally required to have continuous medical and prescription drug coverage for $\geq 12$ months before and $\geq 12$ months after their first (index) cycle of chemotherapy during the index event identification period. Patients receiving biologic agents or targeted therapy alone, in the absence of chemotherapy, were excluded from the study, as were patients with a diagnosis of VTE (ICD-9-CM code 451.1, 451.11, 451.19, 451.2, 451.81, 451.83, 451.84, 451.9, 453.4, 453.41, 453.42, 453.8, 453.9, 997.2, 415.1, $415.11,415.12,415.19)$, severe renal impairment, hemorrhagic stroke, or thrombocytopenia within 12 months before the initial cycle of chemotherapy, and patients with major bleeding (including gastrointestinal or ulcer-related bleeding) within 3 months of the initial cycle of chemotherapy. In addition, patients receiving antithrombotic/thrombolytic treatment, as indicated by pharmacy claims with National Drug Code and Healthcare Common Procedure Coding System codes for anticoagulants, glycoprotein IIb/IIIa receptor antagonists, and thrombolytics, less than 2 weeks before the index cycle of chemotherapy were excluded. Chronic treatment with antiplatelet agents such as aspirin, clopidogrel, or ticlopidine was permitted.

Within the resulting dataset, cancer patients with evidence of a VTE event within 12 months following initiation of chemotherapy, as indicated by the presence of an inpatient or outpatient claim with an ICD-9-CM code for deep vein thrombosis or pulmonary embolism (as listed above for VTE), were identified in accordance with a prior claims study of the incidence of deep vein thrombosis and pulmonary embolism. ${ }^{20}$ For the purposes of cost analysis, additional eligibility criteria were applied, with patients being required to have continuous medical and prescription drug coverage 
for $\geq 12$ months after their first (index) VTE event. Using propensity score methods, ${ }^{23}$ patients with VTE were individually matched to those cancer patients without evidence of a VTE event during this period to form two matched cancer cohorts, ie, VTE and non-VTE (matched control). Each patient with VTE was matched according to propensity score and key patient characteristics, such as cancer site, with three non-VTE patients. Propensity scores were generated using a logistic regression model, with patient cohort (VTE versus non-VTE) as the dependent variable, and age, gender, geographic region, and health plan type as independent variables. Individually matched VTE and non-VTE patients were required to have propensity scores that differed by no more than 0.005 points from each other. If a VTE patient could not be matched with three non-VTE cancer controls, then the VTE patient was excluded from the analysis. The success of propensity score matching was confirmed by the absence of statistically significant differences $(P>0.05)$ in age, gender, geographic region, or health plan type between the resulting VTE and non-VTE patient cohorts.

\section{Patient characteristics and treatment patterns}

Information on patients' demographics (gender, age, US Census Bureau geographic region, health plan type) and clinical characteristics (medical conditions/comorbidities, Charlson Comorbidity Index, ${ }^{24,25}$ initial chemotherapy) were collected for both the VTE and non-VTE (control) cohorts over the 12-month baseline period preceding the index VTE event. For the VTE cohort, the index event was defined as the first VTE diagnosis occurring after the initial cycle of chemotherapy; for the non-VTE cohort, the index event was a random date occurring within the 12-month period following the initial cycle of chemotherapy.

\section{Health care resource utilization and costs}

Resource utilization (number of hospitalizations, days in hospital, outpatient medical services, outpatient prescriptions) and costs (inpatient, outpatient, total payments) were determined for the VTE and non-VTE cancer cohorts over the 12-month period following the index VTE event. Resource usage and costs were determined for all-cause claims (ie, all claims) and VTE-related claims (ie, claims with an associated ICD-9-CM diagnostic code for deep vein thrombosis or pulmonary embolism as defined above). VTE-related hospitalization was defined as a hospitalization that contained a diagnostic code for deep vein thrombosis or pulmonary embolism. The incremental impact of VTE on all-cause and VTE-related health care costs was determined for the entire cancer cohort and separately for patients with bladder, colorectal, lung, ovarian, pancreatic, and gastric cancer. Costs were adjusted using multivariate regression to account for demographic (age, gender, region, health plan) and clinical (VTE type, Charlson Comorbidity Index) covariates. All costs were inflation-adjusted to 2010 levels, using the CPI Medical Care Index.

\section{Statistical analysis}

Intercohort comparisons of patient demographic, clinical, and treatment characteristics, and clinical outcomes were performed using Student's $t$-test. A $P$ value of $<0.05$ was considered statistically significant. Resource utilization and unadjusted health care costs were summarized with descriptive statistics and compared between cohorts using Student's $t$-test. A generalized linear model was fitted to the cost data with a gamma probability distribution and a log link function. Adjusted incremental costs ofVTE were derived from the multivariate regression model. Statistical analyses were conducted using $\mathrm{SAS}^{\circledR}$ version 9.2 (SAS Institute Inc, Cary, NC).

\section{Results}

\section{Patient demographics and clinical characteristics}

A total of 63,453 patients with a diagnosis of bladder, colorectal, lung, ovarian, pancreatic, or gastric cancer, and who were undergoing chemotherapy during the index event identification period, were identified from the IMS/PharMetrics PatientCentric database. Of these, 9352 cancer patients fulfilled the study inclusion criteria and provided a pool of patients for propensity score matching. Within this pool, 912 patients with VTE were identified and matched with 2736 non-VTE (control) cancer patients. The two patient cohorts were well balanced in terms of age (mean 63.3 versus 63.0 years) and gender (both 56\% female) distributions. However, the VTE cohort had more frequent comorbidity than the non-VTE cohort (overall mean CCI score 6.8 versus 5.6; $P<0.0001$ ), including pulmonary and hepatic disease, obesity, and congestive heart failure (Table 1). For both patient cohorts, the most frequent cancer types were lung cancer (37.1\% of patients) and colorectal cancer $(33.0 \%$, Table 1$)$.

\section{All-cause health care resource utilization and costs in cancer patients with and without VTE}

During the post-index follow-up period, cancer patients who experienced VTE had approximately three times as many 
Table I Patient demographic and clinical characteristics

\begin{tabular}{|c|c|c|}
\hline & $\begin{array}{l}\text { VTE cohort } \\
(n=9 \mid 2)\end{array}$ & $\begin{array}{l}\text { Matched } \\
\text { non-VTE cohort } \\
(n=2736)\end{array}$ \\
\hline \multicolumn{3}{|l|}{ Age, years } \\
\hline Mean (SD) & $63.3(11.8)$ & $63.0(11.9)$ \\
\hline \multicolumn{3}{|l|}{ Age group, n (\%) } \\
\hline$<35$ years & II (I.2) & $32(1.2)$ \\
\hline $35-54$ years & $192(21.1)$ & $603(22.0)$ \\
\hline $55-74$ years & $547(60.0)$ & $|63|(59.6)$ \\
\hline$\geq 75$ years & $162(17.8)$ & $470(17.2)$ \\
\hline \multicolumn{3}{|l|}{ Gender, n (\%) } \\
\hline Male & 40 I (44.0) & $1205(44.0)$ \\
\hline Female & $5 I I(56.0)$ & $|53|(56.0)$ \\
\hline \multicolumn{3}{|c|}{ Charlson Comorbidity Index, n (\%) } \\
\hline 0 & $12(1.3)$ & $140(5.1)$ \\
\hline $\mathrm{I}-2$ & $126(13.8)$ & $603(22.0)$ \\
\hline $3-4$ & $164(18.0)$ & $594(21.7)$ \\
\hline$\geq 5$ & $610(66.9)$ & $1399(51.1)$ \\
\hline Overall mean (SD) & $6.8(3.3)^{* * *}$ & $5.6(3.5)$ \\
\hline \multicolumn{3}{|l|}{ Cancer type, n (\%) } \\
\hline Bladder & $69(7.6)$ & $207(7.6)$ \\
\hline Colorectal & $301(33.0)$ & $903(33.0)$ \\
\hline Lung & $338(37.1)$ & $1014(37.1)$ \\
\hline Ovarian & $107(1 \mathrm{I} .7)$ & $321(11.7)$ \\
\hline Pancreatic & $58(6.4)$ & $174(6.4)$ \\
\hline Stomach & $39(4.3)$ & $117(4.3)$ \\
\hline \multicolumn{3}{|l|}{ Comorbidities, n (\%) } \\
\hline Hypertension & $475(52.1)$ & $1372(50.2)$ \\
\hline Stroke/TIA & $61(6.7)$ & $160(5.9)$ \\
\hline Diabetes & $188(20.6)$ & $533(19.5)$ \\
\hline Congestive heart failure & $68(7.5)^{*}$ & $153(5.6)$ \\
\hline Pulmonary diseases & $216(23.7)^{*}$ & $551(20.1)$ \\
\hline Liver diseases & $169(18.5)^{*}$ & $424(15.5)$ \\
\hline Atrial fibrillation/flutter & $54(5.9)$ & $155(5.7)$ \\
\hline Obesity & $60(6.6)^{* *}$ & $100(3.7)$ \\
\hline
\end{tabular}

Notes: $* P<0.05$ versus non-VTE cohort; $* * P<0.001$ versus non-VTE cohort; ***P $<0.000$ I versus non-VTE cohort.

Abbreviations: SD, standard deviation; TIA, transient ischemic attack; VTE, venous thromboembolism.

all-cause hospitalizations (mean 1.38 versus 0.55 per patient; $P<0.0001$ ) and days in hospital (mean 10.19 versus 3.37 per patient; $P<0.0001)$ as cancer patients without VTE. Furthermore, outpatient medical claims (mean 291.44 versus 173.39 per patient; $P<0.0001)$ and outpatient prescription claims (mean 39.97 versus 33.07 per patient; $P<0.0001$ ) were also higher in cancer patients with VTE versus those without VTE. Cancer patients who experienced VTE also incurred significantly higher (unadjusted) overall (all-cause) inpatient costs (mean USD 21,299 versus USD 7459 per patient; $P<0.0001$ ), outpatient medical costs (mean USD 47,091 versus USD 29,901 per patient; $P<0.0001$ ), outpatient prescription costs (mean USD 6569 versus USD 4331 per patient; $P<0.0001$ ) and total health care costs (mean USD 74,959 versus USD 41,691 per patient; $P<0.0001$ ) over the 12-month post-VTE follow-up period than those without VTE (Table 2). The total cost per hospitalization for deep vein thrombosis and pulmonary embolism was (mean) USD 6654 and USD 10,753, respectively.

\section{VTE-related resource utilization and costs in cancer patients with and without VTE}

As anticipated, VTE-related hospitalizations (mean 0.63 versus 0.0 per patient; $P<0.0001$ ) and VTE-related days in hospital (mean 5.8 versus 0.0 per patient; $P<0.0001$ ) were confined exclusively to cancer patients with VTE rather than those without VTE. Likewise, VTE-related outpatient medical claims (mean 23.3 versus 0.0 per patient; $P<0.0001$ ) and outpatient prescription claims (mean 4.8 versus 0.0 per patient; $P<0.0001$ ) were limited to cancer patients with VTE. Cancer patients who experienced VTE incurred (unadjusted) mean VTE-related inpatient costs of USD 5202 per patient, outpatient medical costs of USD 2063 per patient, outpatient prescription costs of USD 1982 per patient, and total VTE-related health care costs of USD 9247 per patient over the 12-month post-VTE follow-up period (Table 2).

\section{Incremental costs of VTE in cancer patients}

After adjusting for demographic and clinical differences between cancer patients with and without VTE, the predicted incremental all-cause health care cost of VTE (compared with no VTE) was (mean) USD 30,538 (standard error [SE] USD 2349) per patient, while the predicted incremental VTE-related health care cost of VTE (compared with no VTE) was (mean) USD 9202 (SE USD 378) per patient (both $P<0.0001$ ) over the 12-month post-index follow-up period. Predicted mean incremental all-cause health care costs of VTE ranged from USD 11,946 per patient for gastric cancer to USD 38,983 per patient for pancreatic cancer (Figure 1). Predicted mean incremental VTE-related health care costs of VTE ranged from USD 4524 per patient for bladder cancer to USD 17,205 per patient for pancreatic cancer (Figure 1).

\section{Discussion}

This population database study demonstrated that high-risk cancer patients initiating contemporary chemotherapy regimens for common solid tumors who experienced VTE had approximately three times as many hospitalizations and days in hospital, and one-third more outpatient claims, than propensity-matched cancer patients without VTE over 
Table 2 All-cause and VTE-related health care resource use and associated costs for cancer patients with and without VTE

\begin{tabular}{|c|c|c|c|c|}
\hline & \multicolumn{2}{|c|}{ VTE cohort $(n=912)$} & \multicolumn{2}{|c|}{ Non-VTE cohort $(n=2736)$} \\
\hline & VTE-related & All-cause & VTE-related & All-cause \\
\hline \multicolumn{5}{|l|}{ Resource utilization, mean (SD) } \\
\hline Hospitalizations/patient (n) & $0.63(0.90)^{*}$ & $1.38(1.67)^{*}$ & $\mathrm{~N} / \mathrm{A}$ & $0.55(\mathrm{I} . \mathrm{II})$ \\
\hline Hospital days/patient (n) & $5.79(15.55)^{*}$ & $10.19(18.98)^{*}$ & $\mathrm{~N} / \mathrm{A}$ & $3.37(10.14)$ \\
\hline Outpatient medical claims/patient (n) & $23.33(43.95)^{*}$ & $291.44(220.33)^{*}$ & $N / A$ & $173.39(166.19)$ \\
\hline Outpatient prescription claims/patient (n) & $4.75(5.78)^{*}$ & $39.97(33.52)^{*}$ & $\mathrm{~N} / \mathrm{A}$ & 33.07 (33.09) \\
\hline \multicolumn{5}{|l|}{ Costs (USD), mean (SD) } \\
\hline Inpatient cost/patient & $5202(15,847)^{*}$ & $21,299(46,872)^{*}$ & $\mathrm{~N} / \mathrm{A}$ & $7459(23,657)$ \\
\hline \multicolumn{5}{|l|}{ Outpatient cost/patient } \\
\hline Medical services & $2063(6247)^{*}$ & $47,091(58,559)^{*}$ & $\mathrm{~N} / \mathrm{A}$ & $29,90 \mathrm{I}(46,545)$ \\
\hline Prescription drugs & $1982(6652)$ & $6569(12,167)^{*}$ & $\mathrm{~N} / \mathrm{A}$ & $4331(9216)$ \\
\hline Overall cost/patient & $9247(19,650)^{*}$ & $74,959(83,302)^{*}$ & $\mathrm{~N} / \mathrm{A}$ & $41,69 \mid(58,502)$ \\
\hline
\end{tabular}

Note: $* P<0.0001$ versus non-VTE cohort.

Abbreviations: N/A, not applicable; SD, standard deviation; USD, US dollars; VTE, venous thromboembolism.

the 12-month post-VTE follow-up period. Total (all-cause) health care costs were about $80 \%$ higher in cancer patients with VTE (mean unadjusted cost USD 74,959 per patient) in comparison with matched cancer patients without VTE (USD 41,691).

However, it should be emphasized that this VTE cost differential could be partly due to clinical differences between the two groups. Patients with VTE had more frequent comorbidity than those without VTE, although some of this might have been due to the complications of VTE itself. The risk of VTE is influenced by factors such as disease stage, treatment modality, and primary cancer site. ${ }^{9,11,15,17,26}$ Pancreatic and gastric cancers are associated with the highest rates of VTE, whereas breast cancers and melanomas show the lowest rates. ${ }^{3,17}$ However, we used propensity matching techniques ${ }^{23}$ to ensure that the VTE and non-VTE cohorts were matched with regard to their type of cancer. Furthermore, generalized linear models were used to adjust for demographic and clinical confounders and thereby avoid this potential bias. After adjusting for these and other covariates through multivariate regression, VTE accounted for $30 \%$ of the overall difference in health care costs between

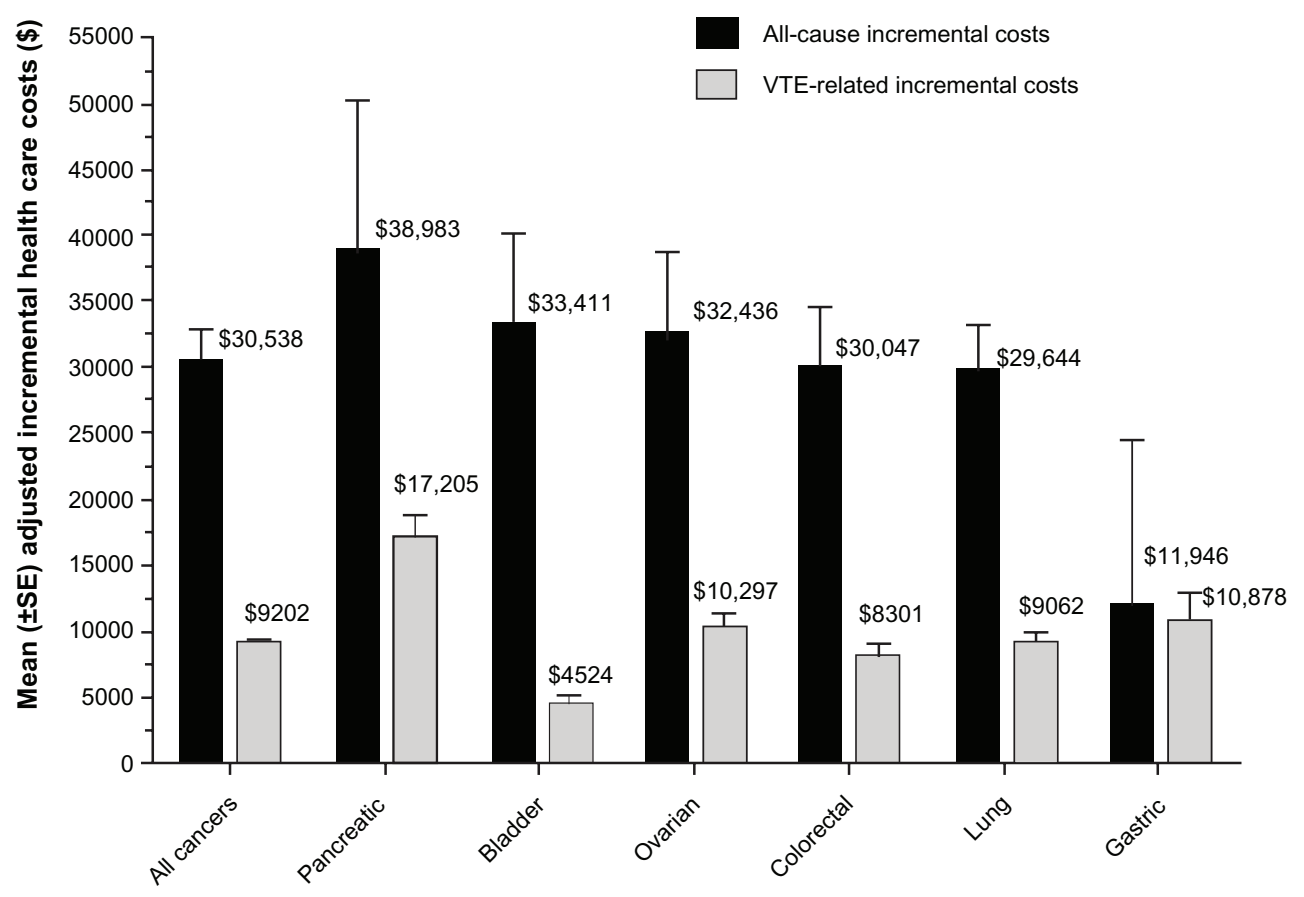

Figure I Adjusted incremental health care costs for cancer patients with and without VTE, presented by site of cancer.

Note: Health care costs are presented from the US payer's perspective, and represent payments made by health plans for services provided. Abbreviations: SE, standard error; VTE, venous thromboembolism. 
cancer patients with and without VTE. This figure varied widely between the various cancer types, ranging from a low of $13.5 \%$ in bladder cancer to a high of $91.1 \%$ in gastric cancer. It should be noted that the estimates of the overall incremental cost of VTE are likely to be inflated through inclusion of the costs of treating VTE-related complications as well as the costs of VTE itself.

The economic burden of VTE is primarily driven by the development of complications (notably bleeding, and, in the longer term, VTE recurrence and post-thrombotic syndrome), and by the length of hospitalization. ${ }^{18}$ Information from the general medical population suggests that the direct medical costs of VTE are large, ${ }^{19,27,28}$ with the annual overall cost of VTE to the health care system in the US estimated to exceed USD 1.5 billion. ${ }^{28} \mathrm{~A}$ large retrospective US database analysis (1997-2004) reported that the median annualized direct medical costs of a primary deep vein thrombosis or pulmonary embolism event in the general population were USD 17,512 and USD 18,901, respectively, rising to USD 20,569 (2004 USD values) when complicated by postthrombotic syndrome. ${ }^{27}$

The high rates of bleeding complications (about 12\%) and VTE recurrence (about 17\%-21\%) among cancer patients with VTE $^{10,18}$ suggest that cancer-associated VTE is likely to be more costly than VTE in the general medical population, although available information is sparse. A retrospective analysis (1992-1994) attributed about 6\% of oncology bed occupancy directly to VTE and VTE-associated complications. ${ }^{21}$ A review of medical records of cancer patients $(n=529)$ presenting with deep vein thrombosis during the 1994-1997 period indicates that the mean length of hospital stay for initiation of anticoagulation was 11 days, and that the mean cost of the index hospitalization was USD 20,065 (2002 USD values). ${ }^{18}$ Our findings suggest that direct medical costs amount, on average, to about USD 75,000 per patient over the first 12 months following a VTE event, and that the adjusted incremental cost associated with VTE in cancer ranges from approximately USD 12,000-39,000 per patient per year, depending on the tumor site. This study provides a contemporary estimate of the economic burden of cancer-associated VTE in the ambulatory setting, where most cancer care occurs.

VTE can be prevented with appropriate pharmacologic and mechanical prophylaxis. Multiple randomized studies have shown the benefit of thromboprophylaxis both in hospitalized patients with acute medical illness, including cancer, and in the surgical setting. ${ }^{29,30}$ However, most cancer care, and therefore most episodes of VTE, occur in the outpatient setting. ${ }^{31}$ Risk factors identified for development of symptomatic chemotherapy-associated VTE in cancer outpatients include: cancer site, prechemotherapy platelet count $\geq 350 \times 10^{9} / \mathrm{L}$, hemoglobin level $<100 \mathrm{~g} / \mathrm{L}$ or use of erythropoiesis-stimulating agents, prechemotherapy leukocyte count $>11 \times 10^{9} / \mathrm{L}$, and body mass index $>35 \mathrm{~kg} / \mathrm{m}, 2,26$ and these factors collectively form the basis of a validated VTE risk scoring system for cancer patients receiving chemotherapy. ${ }^{26,32}$ A number of candidate biomarkers of thrombotic risk in cancer have been proposed, including D-dimer, soluble P-selectin, C-reactive protein, and tissue factor. ${ }^{33-36}$ Addition of D-dimer and soluble P-selectin to the VTE risk scoring system increases its predictive accuracy. ${ }^{32}$ However, D-dimer and P-selectin assays are not routinely performed in cancer patients.

The Current American Society of Clinical Oncology and National Comprehensive Cancer Network guidelines recommend thromboprophylaxis in the outpatient setting only for highly selected cancer patients, particularly multiple myeloma patients receiving thalidomide-based or lenalidomide-based combination regimens. ${ }^{8,37}$ Recent controlled studies demonstrate that pharmacologic anticoagulation is effective and safe in reducing VTE in selected outpatients receiving chemotherapy. These include PROTECHT, a study of nadroparin in patients with solid tumors, ${ }^{38}$ CONKO 004, a study of enoxaparin in pancreatic cancer, ${ }^{39}$ and FRAGEM, a study of dalteparin in pancreatic cancer. ${ }^{40}$ The largest study of thromboprophylaxis, SAVE-ONCO, was a randomized, placebo-controlled trial of the ultralow-molecular-weight heparin, semuloparin, in patients initiating chemotherapy for solid tumors (lung, pancreas, stomach, colon/rectum, bladder, and ovary). ${ }^{41}$ The six common solid tumors included in our analysis were also investigated in the PROTECHT and SAVE-ONCO studies. Our findings suggest that thromboprophylaxis targeted at cancer patients at high risk of VTE can potentially reduce the economic burden of VTE and, hence, overall cancer costs.

As an administrative claims database investigation, our study has several limitations. The IMS/PharMetrics PatientCentric database represents a commercially insured population and may contain biases or have limited applicability to other populations. Information on tumor stage, histologic subtype, and leukocyte and platelet counts, which may influence the risk of bleeding and thrombosis, was not included in the database. Use of claims data also precludes verification of diagnoses and raises the possibility of coding error, given that it was not possible to verify whether diagnoses and procedures were accurately coded. Finally, the predicted 
incremental health care costs of VTE are likely to be conservative estimates, because they do not take into account the cost of VTE complications or the medical costs arising as a result of VTE-imposed interruption of cancer therapy.

\section{Conclusion}

The high numbers of all-cause hospitalizations, days in hospital, and outpatient claims associated with cancer patients with VTE, in comparison with matched cancer patients without VTE, indicate that VTE imposes a substantial clinical and economic burden in the ambulatory oncology setting. In the first 12 months following the index VTE event, all-cause health care costs were, on average, USD 30,538 per patient higher in cancer patients with VTE than in those without VTE, with VTE-related health care costs accounting for approximately one-third of this difference. Measures to prevent cancer-associated VTE in the outpatient setting offer the potential to reduce the high health care utilization and costs of high-risk cancer patients.

\section{Disclosure}

Editorial/writing assistance in the preparation of this paper was provided by Paul Lane and Andrew Fitton of UBC Scientific Solutions and was funded by sanofi-aventis US. AAK, MRD, JL, and GCC were fully responsible for all content and editorial decisions. The authors received no honoraria related to the development of this paper. AAK has acted in a consultant/advisory role for sanofi-aventis, Janssen, Bristol-Myers-Squibb, Daiichi Sankyo, and Roche, has received honoraria from sanofi-aventis, and has received research funding from sanofi-aventis. MRD is an employee of sanofi-aventis US. JL is an employee of Novosys Health, which has received research funding from sanofi-aventis. GCC has no conflicts of interest to disclose. This study was presented in part as Abstract 6059 at the 48th Annual Meeting of the American Society of Clinical Oncology, June 1-5, 2012, Chicago, IL.

\section{References}

1. Noble S, Pasi J. Epidemiology and pathophysiology of cancer-associated thrombosis. Br J Cancer. 2010;102 Suppl 1:S2-S9.

2. Kessler CM. The link between cancer and venous thromboembolism: a review. Am J Clin Oncol. 2009;32:S3-S7.

3. Khorana AA, Connolly GC. Assessing risk of venous thromboembolism in the patient with cancer. J Clin Oncol. 2009;27:4839-4847.

4. Geerts WH, Bergqvist D, Pineo GF, et al. Prevention of venous thromboembolism: American College of Chest Physicians EvidenceBased Clinical Practice Guidelines (8th ed). Chest. 2008;133: 381S-453S.

5. Heit JA, Silverstein MD, Mohr DN, et al. Risk factors for deep vein thrombosis and pulmonary embolism: a population-based case-control study. Arch Intern Med. 2000;160:809-815.
6. Lip GY, Chin BS, Blann AD. Cancer and the prothrombotic state Lancet Oncol. 2002;3:27-34.

7. De Cicco M. The prothrombotic state in cancer: pathogenic mechanisms. Crit Rev Oncol Hematol. 2004;50:187-196.

8. Lyman GH, Khorana AA, Falanga A, et al. American Society of Clinical Oncology guideline: recommendations for venous thromboembolism prophylaxis and treatment in patients with cancer. J Clin Oncol. 2007;25:5490-5505

9. Levitan N, Dowlati A, Remick SC, et al. Rates of initial and recurrent thromboembolic disease among patients with malignancy versus those without malignancy. Risk analysis using Medicare claims data. Medicine (Baltimore). 1999;78:285-291.

10. Prandoni P, Lensing AW, Piccioli A, et al. Recurrent venous thromboembolism and bleeding complications during anticoagulant treatment in patients with cancer and venous thrombosis. Blood. 2002; 100:3484-3488.

11. Sallah S, Wan JY, Nguyen NP. Venous thrombosis in patients with solid tumors: determination of frequency and characteristics. Thromb Haemost. 2002;87:575-579.

12. Khorana AA, Francis CW, Culakova E, et al. Thromboembolism in hospitalized neutropenic cancer patients. J Clin Oncol. 2006;24: 484-490.

13. Blom JW, Doggen CJ, Osanto S, et al. Malignancies, prothrombotic mutations, and the risk of venous thrombosis. JAMA. 2005;293: 715-722.

14. Blom JW, Vanderschoot JP, Oostindier MJ, et al. Incidence of venous thrombosis in a large cohort of 66,329 cancer patients: results of a record linkage study. J Thromb Haemost. 2006;4:529-535.

15. Stein PD, Beemath A, Meyers FA, et al. Incidence of venous thromboembolism in patients hospitalized with cancer. Am J Med. 2006;119: 60-68.

16. Agnelli G, Bolis G, Capussotti L, et al. A clinical outcome-based prospective study on venous thromboembolism after cancer surgery: the @ RISTOS project. Ann Surg. 2006;243:89-95.

17. Wun T, White RH. Epidemiology of cancer-related venous thromboembolism. Best Pract Res Clin Haematol. 2009;22:9-23.

18. Elting LS, Escalante CP, Cooksley C, et al. Outcomes and cost of deep venous thrombosis among patients with cancer. Arch Intern Med. 2004;164:1653-1661.

19. Spyropoulos AC, Lin J. Direct medical costs of venous thromboembolism and subsequent hospital readmission rates: an administrative claims analysis from 30 managed care organizations. J Manag Care Pharm. 2007;13:475-486.

20. Amin AN, Lin J, Yang G, et al. Are there any differences in the clinical and economic outcomes between US cancer patients receiving appropriate or inappropriate venous thromboembolism prophylaxis? J Oncol Pract. 2009;5:159-164.

21. Harrington KJ, Bateman AR, Syrigos KN, et al. Cancer-related thromboembolic disease in patients with solid tumours: a retrospective analysis. Ann Oncol. 1997;8:669-673.

22. Spyropoulos AC, Hussein M, Lin J, et al. Rates of symptomatic venous thromboembolism in US surgical patients: a retrospective administrative database study. J Thromb Thrombolysis. 2009;28:458-464.

23. Baser O. Too much ado about propensity score models? Comparing methods of propensity score matching. Value Health. 2006;9:377-385.

24. Charlson ME, Pompei P, Ales KL, et al. A new method of classifying prognostic comorbidity in longitudinal studies: development and validation. J Chronic Dis. 1987;40:373-383.

25. Quan H, Sundararajan V, Halfon P, et al. Coding algorithms for defining comorbidities in ICD-9-CM and ICD-10 administrative data. Med Care. 2005;43:1130-1139.

26. Khorana AA, Kuderer NM, Culakova E, et al. Development and validation of a predictive model for chemotherapy-associated thrombosis. Blood. 2008;111:4902-4907.

27. MacDougall DA, Feliu AL, Boccuzzi SJ, et al. Economic burden of deep-vein thrombosis, pulmonary embolism, and post-thrombotic syndrome. Am J Health Syst Pharm. 2006;63:S5-S15. 
28. Dobesh PP. Clopidogrel versus prasugrel: times are changing, but not for everyone. Pharmacotherapy. 2009;29:1393-1396.

29. Lee AY. Thrombosis in cancer: an update on prevention, treatment, and survival benefits of anticoagulants. Hematology Am Soc Hematol Educ Program. 2010;2010:144-149.

30. Stanley A, Young A. Primary prevention of venous thromboembolism in medical and surgical oncology patients. Br J Cancer. 2010;102 Suppl 1: S10-S16.

31. Khorana AA, Dalal M, Tangirala K, et al. Higher incidence of venous thromboembolism in the outpatient versus the inpatient setting among US cancer patients. Abstract 674 presented at the 53rd American Society of Hypertension. San Diego, CA, December 10-13, 2011.

32. Ay C, Dunkler D, Marosi C, et al. Prediction of venous thromboembolism in cancer patients. Blood. 2010;116:5377-5382.

33. Ay C, Vormittag R, Dunkler D, et al. D-dimer and prothrombin fragment $1+2$ predict venous thromboembolism in patients with cancer: results from the Vienna Cancer and Thrombosis Study. J Clin Oncol. 2009;27:4124-4129.

34. Ay C, Simanek R, Vormittag R, et al. High plasma levels of soluble $\mathrm{P}$-selectin are predictive of venous thromboembolism in cancer patients: results from the Vienna Cancer and Thrombosis Study (CATS). Blood. 2008;112:2703-2708.

35. Kroger K, Weiland D, Ose C, et al. Risk factors for venous thromboembolic events in cancer patients. Ann Oncol. 2006;17:297-303.
36. Uno K, Homma S, Satoh T, et al. Tissue factor expression as a possible determinant of thromboembolism in ovarian cancer. Br J Cancer. 2007; 96:290-295.

37. National Comprehensive Cancer Network. NCCN clinical practice guidelines in oncology. Venous thromboembolic disease, 2011.

38. Agnelli G, Gussoni G, Bianchini C, et al. Nadroparin for the prevention of thromboembolic events in ambulatory patients with metastatic or locally advanced solid cancer receiving chemotherapy: a randomised, placebo-controlled, double-blind study. Lancet Oncol. 2009;10: 943-949.

39. Riess H, Pelzer U, Deutschinoff G, et al. A prospective, randomized trial of chemotherapy with or without the low molecular weight heparin (LMWH) enoxaparin in patients (pts) with advanced pancreatic cancer (APC): results of the CONKO 004 trial. J Clin Oncol. 2009; 27(Suppl 18):Abstract LBA4506.

40. Maraveyas A, Holmes M, Lofts F, et al. Chemoanticoagulation versus chemotherapy in advanced pancreatic cancer (APC): Results of the interim analysis of the FRAGEM trial. J Clin Oncol. 2007;25(Part I, Suppl 18): Abstract 4583 .

41. Agnelli G, George DJ, Kakkar AK, et al. Semuloparin for thromboprophylaxis in patients receiving chemotherapy for cancer. $N$ Engl J Med. 2012;366:601-609.
ClinicoEconomics and Outcomes Research

\section{Publish your work in this journal}

ClinicoEconomics \& Outcomes Research is an international, peerreviewed open-access journal focusing on Health Technology Assessment, Pharmacoeconomics and Outcomes Research in the areas of diagnosis, medical devices, and clinical, surgical and pharmacological intervention. The economic impact of health policy and health systems

\section{Dovepress}

organization also constitute important areas of coverage. The manuscript management system is completely online and includes a very quick and fair peer-review system, which is all easy to use. Visit http://www.dovepress.com/testimonials.php to read real quotes from published authors. 